CREAT. MATH. INFORM.

Volume 25 (2016), No. 1,

Pages $121-126$

\title{
Approximating fixed points of demicontractive mappings by iterative methods defined as admissible perturbations
}

\author{
CRISTINA ŢICALĂ
}

ABSTRACT. The aim of this paper is to prove some convergence theorems for a general Krasnoselskij type fixed point iterative method defined by means of the concept of admissible perturbation of a demicontractive operator in Hilbert spaces.

\section{INTRODUCTION}

The most important explicit fixed point iterative methods used in literature, see [2], are: Picard iteration (also named sequence of successive approximations), Krasnoselskij iteration, Mann iteration and Ishikawa iteration.

We recall some of the most used such methods, see [2].

Let $E$ be a real vector space and $T: E \rightarrow E$ a given operator. Let $x_{0} \in E$ be arbitrary and $\left\{\alpha_{n}\right\} \subset[0,1]$ a sequence of real numbers. The sequence $\left\{x_{n}\right\}_{n=0}^{\infty} \subset E$ defined by $x_{0} \in E$ and

$$
x_{n+1}=\left(1-\alpha_{n}\right) x_{n}+\alpha_{n} T x_{n}, n=0,1,2 \ldots
$$

is called Mann Algorithm.

The sequence $\left\{x_{n}\right\}_{n=0}^{\infty} \subset E$ defined by

$$
\left\{\begin{array}{rll}
x_{n+1} & =\left(1-\alpha_{n}\right) x_{n}+\alpha_{n} T y_{n}, & n=0,1,2, \ldots \\
y_{n} & =\left(1-\beta_{n}\right) x_{n}+\beta_{n} T x_{n}, & n=0,1,2, \ldots
\end{array} ;\right.
$$

where $\alpha_{n}$ and $\beta_{n}$ are sequences of positive numbers in [0,1], and $x_{0} \in E$ arbitrary, is called Ishikawa algorithm.

Remark 1.1. For $\beta_{n}=0$ (1.2) reduces to (1.1), while for $\alpha_{n}=\lambda$ (constant) the Mann algorithm process (1.1) reduces to the so called Krasnoselskij algorithm ([12]).

$$
x_{n+1}=(1-\lambda) x_{n}+\lambda T x_{n}, n=0,1,2, \ldots
$$

The main intersest with respect to a certain fixed point iterative method is to obtain a convergence theorem for a large class of contractive operators defined as general as possible. For a self mapping $T$ of a compact interval of the real line having an unique fixed point, Mann in [13] proved that the iteration process $x_{n+1}=\left(1-a_{n}\right) x_{n}+a_{n} T x_{n}$ with $a_{n}=\frac{1}{n+1}$ converges to the fixed point. Franks and Marzec [9] proved that the uniqueness requirement was unnecessary. Outlaw and Groetsch [15] generalized the procedure for $T$ - a nonexpansive mapping on a convex, compact subset of the complex plane and then, Groetsch in [10] for uniformly convex Banach spaces.

Hicks and Kubicek in [11] enlarged the class of mappings for which the iteration process converges to a fixed point of demicontractive mappings.

Received: 27.01.2016. In revised form: 29.01.2016. Accepted:

1991 Mathematics Subject Classification. 47H10, 47H09.

Key words and phrases. Fixed point, Krasnoselskij iteration, admissible perturbation. 
Theorem 1.1. [11] Suppose $C$ is a convex subset of $H$. Suppose $T$ is a demicontractive mapping of $C$ into itself with contraction coefficient $k$. Suppose the set of fixed points $F(T)$ is nonempty and $\sum d_{n}\left(1-d_{n}\right)$ diverges and $d_{n} \leftarrow d<1-k$. Then $\lim \inf \left\|v_{n}-T v_{n}\right\|=0$ for each $v_{1} \in C$ where $v_{n+1}$ is defined by

$$
v_{n+1}=\left(1-d_{n}\right) v_{n}+d_{n} T v_{n}
$$

The theory of admissible perturbations of an operator introduced by Rus in [16] opened a new direction of research and unified the most important aspects of the iterative approximation of fixed point for single valued self operators.

Definition 1.1. [16] Let $X$ be a nonempty set. A mapping $G: X \times X \rightarrow X$ is called admissible if it satisfies the following two conditions:

$(A 1) \quad G(x, x)=x, \quad$ for all $\quad x \in X$;

(A2) $\quad G(x, y)=x$ implies $y=x$.

Let $f: X \rightarrow X$ be an operator. Then we consider the operator $f_{G}: X \rightarrow X$ defined by

$$
f_{G}(x):=G(x, f(x)) .
$$

We remark that

$$
F_{f_{G}}=F_{f}:=\{x \in X \mid f(x)=x\} .
$$

Berinde [3], [5] continued the study of fixed point iterative methods by means of the theory of admissible perturbations and obtained very general convergence theorems for Krasnoselskij type fixed point iterative methods defined as admissible perturbations of a nonlinear operator for the class of nonexpansive operators and $\varphi$ - pseudocontractive operators on Hilbert spaces.

Using these results, our aim is to prove some convergence theorems for approximating fixed points of demicontractive mappings.

In this way, we extend the theorems of Hicks and Kubicek by using the admissible perturbation technique for demicontractive mappings for Krasnoselskij iteration.

Definition 1.2. [16] Let $f: X \rightarrow X$ a nonlinear operator and $G: X \times X \rightarrow X$ be an admissible mapping. The iterative algorithm $\left\{x_{n}\right\}$ given by $x_{0} \in X$ and

$$
x_{n+1}=G\left(x_{n}, f\left(x_{n}\right)\right), n \geq 0 .
$$

is called the Krasnoselskij algorithm corresponding to $G$ or the GK-algorithm.

Example 1.1. [16] Let $(V,+, \mathbb{R})$ be a real vector space, $X \subset V$ a convex subset, $\lambda \in(0,1)$, $f: X \rightarrow X$ and $G: X \times X \rightarrow X$ be defined by

$$
G(x, f(x)):=(1-\lambda) x+\lambda f(x), x \in X .
$$

Then $f_{G}$ is admissible perturbation of $f$, which is called the Krasnoselskij perturbation of $f$.

\section{PRELIMINARIES}

Definition 2.3. [16] Let $X$ be a nonempty set. If $f: X \rightarrow X$ is a given mapping and $G: X \times X \rightarrow X$ is an admissible mapping, then the operator $f_{G}: X \rightarrow X$, defined by

$$
f_{G}(x)=G(x, f(x)) ; x \in X ;
$$

is called the admissible perturbation of $f$. 
Definition 2.4. [11] We say that $\mathrm{T}$ is demicontractive if there exists a constant $k<1$ such that, for each fixed point $p$ of $\mathrm{T}$ and each $x \in C$,

$$
\|T x-p\|^{2} \leq\|x-p\|^{2}+k\|x-T x\|^{2} .
$$

We call $k$ the contraction coefficient.

Example 2.2. Define $T$ on $C$ by

$$
T x=\left\{\begin{array}{l}
\frac{2}{3} x \sin \frac{1}{x}, \text { if } x \neq 0 \\
0, \text { if } x=0
\end{array}\right.
$$

This is an example of a demicontractive mapping which is not pseudocontractive, hence not strictly pseudocontractive. Let $C=[-1,1]$. Clearly, 0 is the only fixed point of $T$. Also, for $x \in C,|T x-0|^{2}=|T x|^{2}=\left|\frac{2}{3} x \sin \frac{1}{x}\right| \leq\left|\frac{2 x}{3}\right|^{2} \leq|x|^{2} \leq|x-0|+k|T x-x|^{2}$ for any $k<1$. Thus $T$ is demicontractive.

Let $x=\frac{2}{\pi}$ and $y=\frac{2}{3 \pi}$. Then $|T x-T y|^{2}=\frac{256}{81 \pi^{2}}$. However,

$$
|x-y|^{2}+\left|(I-T) x-(I-T) y \|^{2}=\frac{160}{81 \pi^{2}}<\frac{256}{81 \pi^{2}}=\right| T x-\left.T y\right|^{2} .
$$

Definition 2.5. [2] Let $G: X \times X \rightarrow X$ be an admissible mapping on a normed space $X$. We say that $G$ is affine Lipschitzian if there exists a constant $\mu \in[0,1]$ such that

$$
\left\|G\left(x_{1}, y_{1}\right)-G\left(x_{2}, y_{2}\right)\right\| \leq\left\|\mu\left(x_{1}-x_{2}\right)+(1-\mu)\left(y_{1}-y_{2}\right)\right\|
$$

for all $x_{1}, x_{2}, y_{1}, y_{2} \in X$.

\section{MAIN RESULTS}

The results in this section extend the ones from [11] stated and proved for the Mann iteration to the general case of the admissible perturbation for the Krasnoselskij algorithm.

Theorem 3.2. Let $C$ be a closed, bounded, convex subset of a Hilbert space, H. Suppose $T$ is a demicontractive mapping, $T: C \rightarrow C$ with contraction coefficient $k$. Suppose the set of fixed points $F(T)$ is nonempty.

Suppose $G: C \times C \rightarrow C$ is an affine Lipschitzian admissible mapping with constant $\lambda<1-k$ then $\lim \inf \left\|x_{n}-T x_{n}\right\|=0$ for each $x_{1} \in C$ where $x_{n+1}$ is defined by (1.4).

Proof. We consider the admissible perturbation operator $F: C \times C \rightarrow C$ associated to $T$ and defined by

$$
F x=G(x, T x), x \in C
$$

By the fundamental property of an admissible mapping, see [16], we know that

$$
F i x(F)=F i x(T) \text {. }
$$

Since the admissible mapping, $G$ is affine Lipschitzian, we can take $\mu=\lambda$ and use the fact that $T$ is demicontractive, $T p=p$ and $G(x, x)=x$. We obtain:

$$
\begin{aligned}
\left\|x_{n+1}-p\right\|^{2} & =\left\|F x_{n}-p\right\|^{2}=\left\|G\left(x_{n}, T x_{n}\right)-p\right\|^{2} \\
= & \left\|G\left(x_{n}, T x_{n}\right)-G(p, T p)\right\|^{2} \\
= & \left\|(1-\lambda)\left(x_{n}-p\right)+\lambda\left(T x_{n}-p\right)\right\|^{2} \\
= & \left\|(1-\lambda) x_{n}+\lambda T x_{n}-p\right\|^{2} \\
= & (1-\lambda)\left\|x_{n}-p\right\|^{2}+\lambda\left\|T x_{n}-p\right\|^{2}+\lambda(1-\lambda)\left\|x_{n}-T x_{n}\right\|^{2} \\
\leq & \left\|x_{n}-p\right\|^{2}-\lambda(1-\lambda-k)\left\|x_{n}-T x_{n}\right\|^{2} \\
& \left\|x_{n+1}-p\right\|^{2} \leq\left\|x_{n}-p\right\|^{2}-\lambda(1-\lambda-k)\left\|x_{n}-T x_{n}\right\|^{2}
\end{aligned}
$$


By induction, we obtain

$$
0 \leq\left\|x_{1}-p\right\|^{2}-\lambda(1-\lambda-k) \sum_{j=1}^{n}\left\|x_{j}-T x_{j}\right\|^{2} .
$$

Thus

$$
\lambda(1-\lambda-k) \sum_{n=1}^{\infty}\left\|x_{n}-T x_{n}\right\|^{2} \leq\left\|x_{1}-p\right\|^{2}
$$

This equation shows that $\lim \inf \left\|x_{n}-T x_{n}\right\|=0$

Remark 3.2. In Theorem 3.2 if $\lambda \neq 0$ then we can conclude that $\lim \left\|x_{n}-T x_{n}\right\|=0$.

Remark 3.3. From (3.10) we obtain $\left\|x_{n+1}-p\right\| \leq\left\|x_{n}-p\right\|$, for all $n=0,1,2, \ldots$

Corollary 3.1. Suppose $C$ is a closed, bounded, convex subset of a Hilbert space, H. Suppose T, $\lambda$ and $G$ satisfy the hypothesis of Theorem 3.2. Suppose $I-T$ maps closed bounded subsets of $C$ into closed subsets of $C$ (in particular, if $T$ is demicompact). Then for each $x_{1} \in C$, the iteration process defined by (1.4) converges to a fixed point of $T$.

Corollary 3.2. Suppose $C$ is a closed, bounded, convex subset of a Hilbert space, $H$. Suppose $T$, $\lambda>0$ and $G$ satisfy the hypotesis of remrem 1. Suppose $p$ is a cluster point of $\left\{x_{n}\right\}$ and $T$ is continuous at $p$. Then $x_{n}$ converges to $p$ and $p \in F i x(T)$

Lemma 3.1 (Opial). Suppose $H$ is a Hilbert space and the sequence $\left\{x_{n}\right\}$ is weakly convergent to $x_{0}$. Then for any $x \neq x_{0}$, lim inf $\left\|x_{n}-x_{0}\right\|<\liminf \left\|x_{n}-x\right\|$.

Theorem 3.3. Suppose $C$ is a convex subset of a Hilbert space $H$. Suppose $T: C \rightarrow C$ such that:

(1) $F(T) \neq \emptyset$.

(2) $T$ is demicontractive with contraction coefficient $k$.

(3) If any sequence $v_{n}$ converges weakly to $v$ and $(I-T)\left(v_{n}\right)$ converges strongly to 0 then $(I-T)(v)=0$.

Suppose $G: C \times C \rightarrow C$ is an affine Lipschitzian admissible mapping with constant $\lambda<1-k$.

Then for $x_{1} \in C$ and $\lambda<1-k$, the iteration defined by (1.4) converges weakly to a fixed point of $T$.

Proof. Suppose $T p=p$. By Remark 3.3, we have $\left\|x_{n+1}-p\right\| \leq\left\|x_{n}-p\right\|$ for all $n \geq N$. If $x_{N}=p$ then clearly $x_{n} \rightarrow p$. If $x_{N} \neq p,\left\|x_{N}-p\right\|=r>0$. Let $S_{r}(p)=\{y:\|y-p\| \leq r\}$, and let $D=C \cap S_{r}(p)$. Then $\left\{x_{n}\right\}_{n=N}^{\infty} \subset D$. Also, $D$ is weakly compact since is closed, bounded and convex. Thus there exists a subsequence $\left\{v_{n_{j}}\right\}$ which converges weakly to $y \in D \subset C$. By Remark 3.2, $(I-T) x_{n_{j}} \rightarrow 0$; hence by condition 3, Ty=y so that $G y=y$.

Suppose $\left\{x_{n}\right\}$ does not converge weakly to $y$. Then the sequence $\left\{x_{n}\right\}_{n=N}^{\infty}$ has at least one cluster point $q \neq y$. Suppose $\left\{x_{m_{i}}\right\}$ converges weakly to $q$. As in the case of $y$, $T q=q$ and therefore $G q=q$. From Remark 3.3, we see that the sequences $\left\{\left\|x_{n}-y\right\|\right\}$ and $\left\{\left\|x_{n}-q\right\|\right\}$ are nonincreasing for suficiently large $n$. Thus $\lim \left\|x_{n}-y\right\|$ and $\lim \left\|x_{n}-q\right\|$ both exist. Using Opial's lemma, we obtain the following contradiction:

$$
\begin{aligned}
\lim _{n}\left\|x_{n}-y\right\| & =\liminf _{j}\left\|x_{n_{j}}-y\right\| \\
& <\liminf _{j}\left\|x_{n_{j}}-q\right\| \\
& =\liminf _{i}\left\|x_{m_{i}}-q\right\| \\
& <\liminf _{i}\left\|x_{m_{i}}-y\right\| \\
& =\lim _{n}\left\|x_{n}-y\right\|
\end{aligned}
$$

Therefore, $x_{n}$ converges weakly to $y \in F(T)$ 


\section{NumERICAL EXAMPLE}

Let $C$ be a convex subset of a Hilbert space. Consider the demicontractive mapping given in Example 2.2, i.e., $T: C \rightarrow C$ given by

$$
T x=\left\{\begin{array}{l}
\frac{2}{3} x \sin \frac{1}{x}, \text { if } x \neq 0 \\
0, \text { if } x=0
\end{array}\right.
$$

Now let $C=[-4,4]$ and consider the Krasnoselskij iteraton defined by the Krasnoselskij type admissible perturbation

$$
G(x, y)=(1-\lambda) x+\lambda y, x, y \in X .
$$

Then $T: C \rightarrow C$ satisfies all the assumptions of Theorem 3.3 and $x^{*}=0$ is the unique fixed point of $T$ in $C$. We shall use Maple package to compute the fixed point $x^{*}$ by means of Krasnoselskij iteration, for various values of the parameter $\lambda$ and some initial values $x_{0} \in C$.

Case $1 \lambda=0.5$.

For $x_{0}=4$, after 25 iterations we find the value $x^{*}=3.897899350 \cdot 10^{-9}$.

For $x_{0}=0.1$, after 25 iterations we find the value $x^{*}=6.880966745 \cdot 10^{-11}$.

Case $2 \lambda=0.1$.

For $x_{0}=4$, after 25 iterations we find the value $x^{*}=0.7679623220$.

For $x_{0}=0.1$, after 25 iterations we find the value $x^{*}=0.006063533294$.

Case $3 \lambda=0.9$.

For $x_{0}=4$, after 25 iterations we find the value $x^{*}=-8.463201309 \cdot 10^{-16}$.

For $x_{0}=0.1$, after 25 iterations we find the value $x^{*}-1.326700368 \cdot 10^{-15}$.

In conclusion, as $\lambda$ grows we get faster convergence to the fixed point of $G$.

\section{CONCLUSIONS}

Our Theorem 3.2 involves the GK-algorithm, as it was defined by Rus in [16], which includes as a particular case the Krasnoselskij fixed point iterative algorithm. In the case of the Mann iteration, a similar convergence theorem was obtained in [11] for the same class of mappings.

In the particular case $G(x, T x)=(1-\lambda) x+\lambda T x$ by theorem 3.2 we obtain a convergence theorem for the Krasnoselskij iteration [16].

A more general case for the Mann algorithm associated to $G$ (or the GM-algorithm),

$$
G_{n}\left(x, T x_{n}\right)=\left(1-\lambda_{n}\right) x_{n}-\lambda_{n} T x_{n},
$$

will be studied in a following paper.

For additional informations about different types of operators which were defined as admissible perturbations, see [4], [7], [18] and [19].

\section{REFERENCES}

[1] Berinde, V., Approximating fixed points of Lipschitzian pseudo-contractions, in Mathematics \& mathematics education (Bethlehem, 2000), World Sci. Publishing, River Edge, 2002, NJ, pp. 73-81

[2] Berinde, V., Iterative Approximation of Fixed Points, Lecture Notes in Mathematics, Springer, London, 2007, 2nd edition

[3] Berinde, V., Convergence theorems for fixed point iterative methods defined as admissible perturbations of a nonlinear operator, Carpathian J. Math., 29 (2013), No. 1, 9-18

[4] Berinde, V., Khan, A. R. and Fukhar-ud-din, H., Fixed point iterative methods defined as admissible perturbations of generalized pseudocontractive operators, J. Nonlinear Convex Anal., 16 (2015), No. 3, 563-572

[5] Berinde, V. and Khan, A. R. and Păcurar, M., Convergence theorems for admissible perturbations of $\varphi$ pseudocontractive operators, Miskolc Math. Notes, 15 (2014), 27-37 
[6] Berinde, V. and Kovacs, G., Stabilizing discrete dynamical systems by monotone Krasnoselskij type iterative schemes, Creat. Math. Inform., 17 (2008), 298-307

[7] Berinde, V., Măruşter, Şt., and Rus, I. A., An abstract point of view on iterative approximation pf fixed points of nonself operators, J. Nonlinear Anal., 15 (2014), No. 5, 851-865

[8] Chidume, C., Geometric Properties of Banach Spaces and Nonlinear Iteration, Lecture Notes in Mathematics, 1965, Springer, London, 2009

[9] Franks, R. L. and Marzec, R. P., A theorem on mean-value iterations, Proc. Amer. Math. Soc., 30 (1971), 324-326

[10] Groetsch, C. W., A note on segmenting Mann iterates, J. Math. Anal. Appl., 40 (1972), 369-372

[11] Hicks, T. L. and Kubicek, J. D., On the Mann iteration in a Hilbert space, J. Math. Anal. Appl., 59 (1977), 498-504

[12] Krasnoselskij, M. A., Two remarks on the method of successive approximations (in Russian), Uspehi Mat. Nauk., 10 (1955), No. 1 (63), 123-127

[13] Mann, W. R., Mean value methods in iterations, Proc. Amer. Math. Soc, textbf4 (1953), 506-510

[14] Marinescu, D. S. and Monea, M., Some remarks on the Krasnoselskij iterative method, Creat. Math. Inform., 22 (2013), No. 2, 199-206

[15] Outlaw, C. and Groetsch, C. W., Averaging iteration in a Banach space, Bull. Amer. Math. Soc., 75 (1969), 430-432

[16] Rus, I. A., An abstract point of view on iterative approximation of fixed points, Fixed Point Theory, 13 (2012), 179-192

[17] Rus, I. A. Fixed point theory, Cluj Univ, Press, Cluj-Napoca, 2006

[18] Ţicală, C., Approximating solutions of generalized pseudocontractive variational inequalities by admissible perturbation type iterative methods, Creat. Math. Inform., 22 (2013) No. 2, 237-241

[19] Ţicală, C., A weak convergence theorem for a Krasnoselskij type fixed point iterative method in Hilbert spaces using an admissible perturbation, Sci. Stud. Res. Ser. Math. Inform., 25 (2015), No. 1, 243-252

Department of Mathematics ANd COMPUTER SCIENCE

NORTH UNIVERSity CENTER AT BAIA MARE

TECHNICAL UNIVERSITY OF CLUJ-NAPOCA

VictorieI 76, 430122 BAIA MARE, ROMANIA

Email address: cristina.ticala.popegmail.com 